Revue

de |'histoire des religions

\section{Revue de l'histoire des religions}

$3 \mid 2009$

La culture gallicane. Références et modèles (droit, ecclésiologie, histoire)

\title{
Simon Vigor face aux catholiques zélés : le gallicanisme radical du début du XVII ${ }^{\mathrm{e}}$ siècle
}

Simon Vigor vs. the Catholiques zélés: Radical Gallicanism in the Early 17 th C

Sylvio Hermann De Franceschi

\section{OpenEdition}

Journals

Édition électronique

URL : http://journals.openedition.org/rhr/7279

DOI : $10.4000 /$ rhr.7279

ISSN : 2105-2573

Éditeur

Armand Colin

Édition imprimée

Date de publication : 1 juillet 2009

Pagination : 467-483

ISBN : 978-2200-92591-8

ISSN : 0035-1423

Référence électronique

Sylvio Hermann De Franceschi, « Simon Vigor face aux catholiques zélés : le gallicanisme radical du début du XVII siècle », Revue de l'histoire des religions [En ligne], 3 | 2009, mis en ligne le 01 juillet 2012 consulté le 30 avril 2019. URL : http://journals.openedition.org/rhr/7279 ; DOI : 10.4000/rhr.7279 


\section{Simon Vigor face aux catholiques zélés Le gallicanisme radical du début du $\mathrm{XVII}^{\mathrm{e}}$ siècle}

Le présent article analyse la position du juriste et magistrat Simon Vigor (1556-1624) à l'égard de la doctrine romaine et théocratique, telle que le cardinal Bellarmin l'avait en son temps formulée dans ses Disputationes de controversiis christianae fidei (1586-1593) et dans son De potestate Summi Pontificis in rebus temporalibus (1610). En suivant les théories d'Edmond Richer, l'auteur fameux d'un De ecclesiastica et politica potestate liber unus (1611) qui avait provoqué un immense scandale lors de sa publication, Simon Vigor défend en définitive un gallicanisme radical qui rappelle le De Republica ecclesiastica de Marc'Antonio De Dominis, archevêque apostat réfugié à la cour du roi Jacques $I^{e r}$ d'Angleterre.

\section{Simon Vigor vs. the Catholiques zélés : Radical Gallicanism in the Early 17th $C$.}

This article analyzes the position of the jurist and magistrate Simon Vigor (1556-1624) regarding Roman and theocratic doctrine as formulated by Cardinal Bellarmin in his Disputationes de controversiis christianae fidei (1586-1593) and in his De potestate Summi Pontificis in rebus temporalibus (1610). Following the theories of Edmond Richer, the famous author of the De ecclesiastica et politica potestate liber unus (1611) that had provoked a tremendous scandal when it was published, Simon Vigor ultimately defends a radical Gallicanism reminiscent of the De Republica ecclesiastica of Marc'Antonio De Dominis, an apostate archbishop who had fled to the court of James I of England. 
L'âge posttridentin a été marqué en catholicité par une forte reviviscence de l'antiromanisme. Appuyée sur les acquis du concile, dont les pères lui avaient remis mission d'appliquer et d'interpréter dans l'Église les canons, rassurée par la conclusion des conflits religieux de la seconde moitié du XVI ${ }^{\mathrm{e}}$ siècle, la papauté doit pourtant faire face à des ennemis qui proviennent de sa propre sphère confessionnelle. Parmi eux, les gallicans se signalent à son attention par une exceptionnelle virulence qui redouble au lendemain de la promulgation de l'Édit de Nantes ${ }^{1}$. Il convient de souligner d'emblée le fait que le gallicanisme n'est pas un monolithisme partisan et qu'il ne fait pas que s'intéresser aux seuls rapports entre pouvoir civil et puissance ecclésiale; il est aussi une ecclésiologie, au sens le plus catholique du terme, soit un enseignement qui élabore une conception originale de l'Église sans renier son appartenance confes-

1. Sur le gallicanisme à la fin du XVI $I^{\mathrm{e}}$ siècle et au début du XVII ${ }^{\mathrm{e}}$ siècle, outre les travaux anciens mais classiques de Victor Martin, Le gallicanisme et la réforme catholique. Essai historique sur l'introduction en France des décrets du concile de Trente (1563-1615), Paris, 1919, et id., Le gallicanisme politique et le clergé de France, Paris, 1929, voir Alain Tallon, Conscience nationale et sentiment religieux en France au XVI siècle. Essai sur la vision gallicane du monde, Paris, 2002, et Françoise Hildesheimer, «Entre droit et théologie. L'absolutisme gallican », Les cours d'Espagne et de France au XVII siècle, éd. Chantal Grell et Benoît Pellistrandi, Collection de la Casa de Velázquez, 98, 2007, p. 263-278. On se permet également de renvoyer à Sylvio De Franceschi, «La genèse française du catholicisme d'État et son aboutissement au début du ministériat de Richelieu : les catholiques zélés à l'épreuve de l'affaire Santarelli et la clôture de la controverse autour du pouvoir pontifical au temporel (1626-1627)», Annuaire-bulletin de la Société de l'Histoire de France, Année 2001, p. 19-63, id., « Les théologiens parisiens face à un antiromanisme catholique extrême au temps du richérisme. La condamnation du De Republica ecclesiastica de Marc'Antonio De Dominis par la Sorbonne (15 décembre 1617) ", Chrétiens et sociétés, XVI -XXe siècles. Bulletin du Centre André Latreille (Université Lumière-Lyon 2) et de l'Institut d'Histoire du Christianisme (Université Jean Moulin-Lyon 3), 11, 2004, p. 11-32, id., « Entre aspirations chrétientaires et reconnaissance de l'absolutisme : le sacre du catholicisme d'État. Les catholiques d'État en France et leur tentative de sauvegarde d'un ordre de Chrétienté à l'âge pré-absolutiste : la rupture du ministériat de Richelieu », Stratégies de la Contre-Réforme en France et en Pologne, Actes du Colloque polono-français, Versailles, 26-29 octobre 2000, éd. Ch. Grell, M. Serwański et I. Kraszewski, Poznań, 2006, p. 103-137, et id., « Gallicanisme, antirichérisme et reconnaissance de la romanité ecclésiale : la dispute entre le cardinal Bellarmin et le théologien parisien André Duval (1614)», Papes, princes et savants dans l'Europe moderne. Mélanges à la mémoire de Bruno Neveu, éd. J.-L. Quantin et J.-Cl. Waquet, Genève, 2007, p. 97-121. 
sionnelle. Au moment où se met en place en Europe une modernité politique fondée sur une autonomisation de la légitimité de l'État qui vaut appétence d'absolutisme, l'antiromanisme catholique rencontre la controverse autour du pouvoir pontifical au temporel. La thèse de la potestas indirecta, soit le droit, pour le pontife romain, d'intervenir in rebus temporalibus pour autant que les intérêts du spirituel le requièrent, est exposée pour la première fois sous sa forme classique par le jésuite Robert Bellarmin (1542-1621) dans ses Disputationes de controuersiis christiance fidei (1586-1593). L'ouvrage a retenu l'attention davantage de la curie romaine, soucieuse de ne pas exclure définitivement la théorie théocratique du pouvoir direct, que des gallicans.

L'agressivité des catholiques antiromains a été ravivée par l'essor d'une gigantesque polémique dont les origines factuelles s'ancrent dans le scandale provoqué par l'affaire de l'Interdit vénitien (16061607), à quoi viennent s'ajouter les débats engendrés par l'imposition en 1606 d'un serment d'allégeance aux catholiques anglais, profession de loyauté politique qu'exige Jacques Ir d'Angleterre après l'échec de la Conspiration des Poudres. Troisième événement qui réveille définitivement l'hostilité gallicane : l'assassinat d'Henri IV le 14 mai 1610. Prenant acte des prétentions de la curie romaine, les gallicans entreprennent une offensive de longue haleine, ponctuée par les interventions du Parlement de Paris et soutenue par l'avocat général du roi Louis Servin (1555-1626) et ses affidés ${ }^{2}$. Les parlementaires parisiens prohibent notamment le 26 novembre 1610 la vente et la diffusion du traité De potestate Summi Pontificis in rebus temporalibus (1610) de Bellarmin - était visée la doctrine du pouvoir indirect au motif qu'elle mettait en péril la sécurité du prince.

2. Pour une présentation des événements, voir les ouvrages classiques de Salvo Mastellone, La reggenza di Maria de' Medici, préf. R. Mousnier, Florence, 1962, et de Roland Mousnier, L'Assassinat d'Henri IV (14 mai 1610). Le problème du tyrannicide et l'affermissement de la monarchie absolue, Paris, 1964. Pour une mise en perspective historique de la théorie du pouvoir indirect, consulter S. De Franceschi, "L'autorité pontificale face au legs de l'antiromanisme catholique et régaliste des Lumières : réminiscences doctrinales de Bellarmin et de Suárez dans la théologie politique et l'ecclésiologie catholiques de la mi-XVIII ${ }^{\mathrm{e}}$ siècle à la mi-XIX ${ }^{e}$ siècle », Archivum Historice Pontificice, 38, 2000, p. 119-163, et id., " Le pouvoir indirect du pape au temporel et l'antiromanisme catholique des âges préinfaillibiliste et infaillibiliste : références doctrinales à Bellarmin et à Suárez dans la théologie politique et l'ecclésiologie catholiques du début du XIX ${ }^{\mathrm{e}}$ siècle à la mi$\mathrm{Xx}^{\mathrm{e}}$ siècle ", Revue d'Histoire de l'Eglise de France, t. 88, 2002, p. 103-149. 


\section{L'EXTRÉMISME ANTIROMAIN ET L'ABSOLUTISME PONTIFICAL}

Si la curie romaine n'a pas manqué de défenseurs pour essayer de conforter ses prétentions, il s'est également trouvé nombre d'auteurs remarquables pour signifier au Saint-Siège une brutale fin de non-recevoir. Au temps de l'Interdit vénitien, le servite Paolo Sarpi (1552-1623) s'était fait le chantre du juridictionalisme de la Sérénissime, s'attirant par là les sympathies des gallicans ${ }^{3}$. À la fin de l'année 1611, le propre syndic de la Faculté de théologie de Paris, Edmond Richer (1559-1631)4, faisait paraître son célèbre De ecclesiastica et politica potestate liber unus, qui proposait une nouvelle récapitulation de la doctrine gallicane. À sa suite, Simon Vigor (1556-1624), conseiller au Grand Conseil, publiait successivement en 1613 un Commentarius ex responsione synodali ${ }^{5}$, en 1615 une Apologia de suprema Ecclesice auctoritate ${ }^{6}$, qui s'en prenait à la Disputatio de suprema Romani Pontificis in Ecclesiam potestate (1614) du théologien catholique zélé André Duval (15641638) ${ }^{7}$, et en 1621 les Quatre livres de l'Estat et gouvernement de l'Église $e^{8}$ - Vigor s'y affirmait comme intransigeamment

3. Sur Sarpi, voir Federico Chabod, La politica di Paolo Sarpi, Rome, 1952, et Vittorio Frajese, Sarpi scettico. Stato e Chiesa a Venezia tra cinque e seicento, Bologne, 1994. Consulter aussi S. De Franceschi, «Les irrémédiables brisures de la Chrétienté de l'histoire. Paolo Sarpi entre idée italienne et idéal chrétientaire », Le sentiment national dans l'Europe méridionale aux XVI et XVII siècles (France, Espagne, Italie), éd. A. Tallon, Madrid, 2007, p. 273-293.

4. Sur Richer, voir Monique Cottret, «Edmond Richer (1559-1631): le politique et le sacré », L'État baroque, 1610-1652. Regards sur la pensée politique de la France du premier XVII siècle, dir. H. Méchoulan, préf. A. Robinet, Paris, 1985, p. 159-177, et Claude Sutto, «Une controverse ecclésiologique au début du XVII ${ }^{\mathrm{e}}$ siècle : le Libellus de ecclesiastica et politica potestate d'Edmond Richer (1611)», Homo religiosus. Autour de Jean Delumeau, Paris, 1997, p. $425-430$.

5. S. Vigor, Ex responsione synodali data Basilice oratoribus D. Eugenii Papce IV in congregatione generali III. non. septembr. MCDXXXII de auctoritate cuiuslibet concilii generalis supra Papam et quoslibet fideles pars prcecipua et in eam commentarius, Cologne, 1613.

6. Id., Apologia de suprema Ecclesice auctoritate aduersus Andrceam Duval, doctorem et professorem theologice, Troyes, 1615.

7. A. Duval, De suprema Romani Pontificis in Ecclesiam potestate disputatio quadripartita, Paris, 1614.

8. S. Vigor, Quatre livres de l'Estat et gouvernement de l'Eglise composés par $M^{e}$ Simon Vigor. Préface contenant une sommaire response au livre de $M^{e}$ Theophraste Bouju, Troyes, 1621. 
gallican ${ }^{9}$; il y défendait une doctrine clairement antiromaine et favorable à une stricte séparation des deux sphères d'exercice des puissances temporelle et spirituelle. Entre-temps, l'archevêque apostat de Spalato, Marc'Antonio De Dominis (1560-1624) ${ }^{10}$, qui s'était réfugié en Angleterre auprès de Jacques I ${ }^{\text {er }}$, avait fait paraître les deux premiers tomes de ses dix livres De Republica ecclesiastica, en 1617 et 1620 respectivement - il y prônait une ecclésiologie froidement antiromaine susceptible de séduire à la fois les gallicans et les tenants vénitiens du républicanisme sarpien.

Le débat lancé par le richérisme portait décidément sur la notion de monarchie absolue dans l'Église. Défenseur et disciple fervent de l'ancien syndic destitué par ses collègues de la Faculté de théologie de Paris le $1^{\text {er }}$ septembre 1612, Vigor publie son Commentarius ex responsione synodali pour justifier les thèses du De ecclesiastica et politica potestate. Le gallican revient pesamment sur l'idée selon quoi l'Église est une monarchie tempérée d'aristocratie. Il rappelle qu'il y a deux sortes de puissance monarchique, l'une absolue, quand le peuple a remis l'ensemble de ses droits à un seul, et l'autre tempérée par des limitations qui entravent un exercice déréglé de l'autorité ${ }^{11}$. L'Empereur n'est pas moins monarque parce qu'il doit obtenir l'aval de la Diète pour lever de nouveaux impôts; de même, le pape, qui ne peut révoquer ad libitum canons et décrets conciliaires. Vigor s'emporte contre les théologiens antirichéristes qui veulent faire du souverain pontife un potentat absolu ${ }^{12}$. De rien ne sert, assure Vigor, de se valoir de fallacieuses analogies entre pouvoir civil et puissance ecclésiale - le prince temporel a, certes, droit de vie et de mort sur ses sujets, mais le pape n'est pas maître de prononcer le trépas spirituel

9. Sur Simon Vigor, voir Frédéric Gabriel, «Une réponse aux artifices de parole : François Desmaret sur les pas de Simon Vigor », Stratégies de l'équivoque, Cahiers du Centre de recherches historiques, 33, 2004, p. 175-185.

10. Sur De Dominis, voir Eleonora Belligni, Auctoritas e potestas. Marcantonio De Dominis fra l'Inquisizione e Giacomo I, Milan, 2003.

11. S. Vigor, Commentarius ex responsione synodali, op. cit., $\mathrm{f}^{\mathrm{o}} 21 \mathrm{r}^{\mathrm{o}}$ : «Vna absoluta, cum populus omne ius suum in unum transtulit; altera, cum certis legibus is qui monarcha dicitur est obnoxius ne monarcha omnia pro libidine faciat. "

12. Ibid., $\mathrm{f}^{\mathrm{0}} 21 \mathrm{r}^{\circ}-\mathrm{v}^{\mathrm{o}}$ : "Isti doctores affirmant, et tamen Richerium reprehendunt, quod dixerit monarchiam aristocratico regimine temperatam, et tentant persuadere Papam esse monarcham Ecclesice absolutum et tamen decretis Ecclesice obnoxium, quo nihil magis ridiculum excogitari potest. » 
d'une âme ${ }^{13}$. De surcroît, puissance a été donnée au pape in aedificationem, non pas in destructionem. Le pontife romain n'est pas seulement propter Ecclesiam, il est aussi per Ecclesiam. Le concile était conséquemment supérieur au pape; d'infaillibilité personnelle gratia muneris du souverain pontife, Vigor ne voulait pas entendre parler. Le chef essentiel de l'Église était le Christ, le pape n'en était que le ministériel, soit une tete secondaire : le vicaire ne pouvait être identifié à l'éminente personne qu'il représentait ici-bas.

La diatribe vigorienne était un long plaidoyer en faveur des thèses richéristes qu'elle reprenait entièrement à son compte. Les deux auteurs, Richer et Vigor, étaient désormais unis dans un même combat pour lutter contre les indécentes revendications du SaintSiège en matière temporelle. Dès 1613, Théophraste Bouju leur répliquait dans ses Deux advis ${ }^{14}$. De Bouju, la postérité n'a pratiquement rien retenu si ce n'est son Corps de toute la philosophie divisé en deux parties par démonstration et auctorité d'Aristote, avec esclaircissement de sa doctrine par luy-mesme (1614). L'homme a pourtant été un ardent adversaire du richérisme. Ancien calviniste, converti au catholicisme, Bouju relève d'emblée les implications politiques de la doctrine de Richer : « Nous remarquons parmy ces escrits qui attaquent la monarchie spirituelle de l'Église quelques propositions qui sont directement contre le gouvernement de cet $E_{\text {Estat }}{ }^{15}$. » Contre Richer, Bouju refuse que la puissance civile puisse résider originellement entre les mains des sujets; il se présente assurément absolutiste :

« Richer dit que la puissance coactive des princes dépend du peuple selon la loy divine et de nature, contre laquelle ny l'espace des temps, ny les privilèges des lieux, ny les dignitéz des personnes ne peuvent jamais prescrire. Or encores que cela soit faulx (comme il paroist en l'authorité du père de famille, laquelle ne dépend que de luy et est la première monarchie), néantmoins c'est donner ouverture au peuple de se

13. Ibid., $\mathrm{f}^{\mathrm{o}} 21 \mathrm{v}^{\circ}$ : «Pontifex non habet potestatem absolutam uitce et necis spiritualis in Christianos, propterea quod usus clauium regulatur secundum canones, non autem mera et absoluta potestate exercetur. Nec omnis qui excommunicatur a Papa excommunicatus est in calo. "

14. T. Bouju, Deux advis, l'un sur le livre de M. Edmond Richer intitulé De la puissance ecclésiastique et politique, l'autre sur un livre dont l'autheur ne se nomme point, qui est intitulé Commentaire de l'auctorité de quelque concile général que ce soit sur le pape. De la responce synodale donnée à Basle, Paris, 1613.

15. Ibid., p. II. 
faire accroire que les Estats du royaume assembléz ont plus d'authorité qu'on n'a accoustumé de leur en donner ${ }^{16}$. »

La thèse richériste de la supériorité intrinsèque du régime aristocratique sur le monarchique ne trouvait pas davantage grâce aux yeux de Bouju - là encore, l'argumentation faisait du richérisme un danger pour l'absolutisme de la monarchie française :

«Richer dit que le régime aristocratique est le meilleur de tous et le plus convenable à la nature. Qui doute que si cela estoit persuadé au peuple qu'il ne désirast ce qu'il estimeroit luy estre meilleur? Puisque naturellement chacun tend à son bien, et qu'il n'est pas possible à l'homme de ne désirer pas d'estre heureux ${ }^{17}$. »

La même critique est portée à l'encontre de Simon Vigor, pris en flagrant délit d'anti-absolutisme. Bouju revient sur la définition proposée par le Commentarius d'un régime absolutiste : « Le monarque absolu a puissance de la vie et de la mort sur ses subjects : ce qui luy plaist a vigueur de loix, et encores qu'il prononçast contre les loix, cela est reputé pour droict ${ }^{18}$. » Bouju affirme nettement que tel n'est pas le cas du pontife romain. Il est ensuite extrêmement gêné par le fait que Vigor présente la monarchie française comme purement et absolument monarchique. Du coup, à l'en croire, le Commentarius fait du roi de France le détenteur d'une puissance in destructionem, ce que Bouju estime erroné et contraire à la réalité :

« Les François ne doubtent point que la monarchie de cet Estat ne soit absoluë, mais c'est en la sorte qu'Aristote définit la vraye monarchie, à sçavoir quand la puissance de toutes choses est entre les mains d'une seule personne laquelle imite en son gouvernement la manière d'administrer que tient le père de famille en sa maison ${ }^{19}$. '

De nulle valeur, également, l'argument dévoyé de la transmission de l'autorité par le peuple au souverain - Bouju ne met pas en cause la vérité de la thèse, il s'en prend seulement à l'interprétation qu'en fait Vigor, une fois de plus dénoncé pour anti-absolutisme :

«Qui doute que le peuple n'ait transféré tout son droit au Roy, de quoy il s'ensuit que la souveraine puissance de Sa Majesté est de droit et justice, mais non que le peuple face ce que le Roy face, cela est ridicule. Le peuple est régi et le Roy le régit; autre est l'action du chef qui

16. Ibid., p. 33.

17. Ibid., p. 34.

18. Ibid., p. 18.

19. Ibid., p. 19. 
commande et des membres qui obéissent; ils ne font pas ce qu'il faict, mais ils doivent obéir à ce qu'il commande ${ }^{20}$. »

La proposition valait également dans l'Église comme dans l'État. Qui soutient le contraire est fauteur de troubles dans le royaume aussi bien qu'en catholicité : « Il est aysé à juger par cet échantillon de l'autheur s'il est meilleur françois que catholique, et si sa procédure n'est pas propre à broüiller la monarchie du royaume aussi bien que celle de l'Église pour rendre l'une et l'autre odieuse ${ }^{21}$. » Pas plus que Vigor, Bouju n'est partisan d'un absolutisme déréglé, mais il se refuse à adopter des positions anti-absolutistes. Pour lui, le régime ecclésial est une monarchie absolue, soit un système régi par un monarque de droit divin qui respecte lois et canons.

\section{LES CONSÉQUENCES DOCTRINALES DU RICHÉRISME}

Il semble que le richérisme ait fait imprévisiblement faire au gallicanisme un pas qu'il s'était jusque-là prudemment gardé de franchir mais que Vigor lui a audacieusement emboîté. Publiée en 1615, 1'Apologia de suprema Ecclesice auctoritate récidivait en développant outre mesure les arguments du Commentarius. Ulcéré, Bouju reprend la plume pour rédiger sa Défense pour la hiérarchie de l'Église ${ }^{22}$. Vigor n'y est plus seulement tancé pour condamnable antiabsolutisme; il est expressément qualifié d'hérétique. Dans la polémique, la note gravissime n'est jamais légèrement mentionnée - elle signifie ici que le gallicanisme de Vigor n'est plus l'antiromanisme classique des parlementaires parisiens et de la tradition gallicane qu'ils s'efforçaient de consolider et d'illustrer. Bouju admet de prime abord qu'il est courant de voir le pape attaqué par les hérétiques, mais

« quand on veoid des personnes portantes le nom de catholiques qui tirent contre cette roche et veulent abbattre la hiérarchie de l’Église, on ne peut juger sinon qu'elles ne sçavent pas ce qu'elles font, ou qu'elles ont quelque chose de maling en l'âme contre la religion catholique, qu'elles essayent d'exécuter en cachette, se couvrant de son manteau et en se disant estre de ses enfans, pour desguiser leur mauvais desseing ${ }^{23}$. »

20. Ibid., p. 20.

21. Ibid., p. 20.

22. ID., Deffence pour la hiérarchie de l'Église et de nostre Sainct Père le pape contre les faussetéz et calomnies de maistre Simon Vigor, Paris, 1620.

23. Ibid., p. 1-2. 
Bouju récapitule ensuite les principaux points d'une ecclésiologie orthodoxe. Le Christ est chef essentiel invisible de l'Église triomphante en Purgatoire et militante; le pape, son vicaire, en est le chef essentiel visible ici-bas. Le régime ecclésial est monarchique; il respecte une hiérarchie clairement définie. La primauté de Pierre, et donc de ses successeurs, est indéniable. Le pape exerce la puissance de l'Église lorsqu'elle n'est pas assemblée en corps. Les catholiques, enfin, sont tenus de respecter les décisions du pontife romain ratione fidei et morum. Bouju reconnait cependant que la question de son infaillibilité n'a pas été tranchée :

« Encores que les catholiques ne tiennent pas de certitude de foy que le pape soit infaillible ès jugements des choses qu'il ordonne de sa chaire et fait publier pour estre observées par les catholiques, parce que ceste question n'a point encore esté décidée par l'Église en corps et que Sa Saincteté mesme ne l'a pas déterminée de sa chaire, néantmoins ils le tiennent si certain qu'il n'y peut avoir d'erreur ${ }^{24}$. »

À défaut d'infaillibilité personnelle, il y a une indéfectibilité excathédrale du souverain pontife. Le pape assure l'unité de l'Église; la romanité s'ajoute implicitement aux quatre notes classiques du schéma constantinopolitain, une, sainte, catholique et apostolique $^{25}$. Selon le catholique assurément zélé, le richérisme fait un faux procès à la papauté en l'accusant de viser à un absolutisme tyrannique. Pour Bouju, les fidèles de la catholicité «n'ont jamais attribué davantage de puissance aux Papes ès choses spirituelles que celles d'un monarque tel que nous l'avons descrit, et non tyran, ès choses temporelles $»^{26}$. Quand le Saint Père revendique un pouvoir absolu, il signifie par là qu'il ne dépend que de Dieu. Bouju affirme que Vigor désire rendre en horreur le pape et la monarchie de l'Église - signe incontestable d'hérésie : «N'est-ce pas suivre en ce point l'esprit de Luther et de Calvin et des autres hérétiques en se disant catholique ${ }^{27}$ ? » Luthéranisme et wycliffisme sont les deux sources à quoi Vigor a puisé - telle est la conviction de Bouju,

24. Ibid., p. 13.

25. Voir S. De Franceschi, «Approches de la romanité ecclésiale du concile de Trente au Syllabus. L'idée romaine dans la définition de l'Église : parcours d'une interrogation critique ", L'idée de Rome : pouvoirs, représentations, conflits. Actes de la XII Université d'été d'histoire religieuse, Rome, 10-15 juillet 2003, éd. H. Multon et C. Sorrel, Chambéry, 2006, p. 47-65.

26. T. Bouju, op. cit., p. 22.

27. Ibid., p. 23. 
qui emprunte un chemin que les adversaires de Paolo Sarpi et de De Dominis avaient déjà parcouru :

«Si Vigor ne croit pas que le régime de l'Église soit monarchique, que ne le dit-il aussi franchement comme les hérétiques qu'il suit, car Illiricus ne feint point de dire qu'il est démocratique, et Luther, et Bèze après eux les suivant, qu'il est aristocratique, et Wiclef nioit qu'il y eust un chef visible icy bas en l'Église ${ }^{28}$. »

Le débat sur la primauté pétrine est encore une fois l'occasion à Bouju de taxer Vigor d'hérésie, et désormais la calviniste s'ajoute à la liste précédente. Pour Bouju, la potentior principalitas de la Sedes Petri et du sedens est condition d'unité ecclésiale :

«Nous concluons de tout ce que dessus que ce que Calvin et Vigor après luy mettent en avant est faux, à sçavoir que sainct Pierre n'avoit que le premier lieu d'honneur et que s'il estoit vraye, l'Église ne pourroit subsister, pour ce que si le Pape n'avoit jurisdiction sur toute l'Église catholique esparse en plusieurs provinces et divers patriarchats, il seroit impossible de la maintenir en unité, car les Estats non seulement aristocratiques, mais mesmes les populaires, quelques petits qu'ils soyent, ont besoing de réduire leur auctorité en unité, comme si c'estoit une monarchie ${ }^{29}$. »

Les indices se multipliaient d'une héréticité de la doctrine vigorienne. Même le conciliarisme, certes condamné par le SaintSiège, mais illustré par une longue série de docteurs tenus unanimement pour catholiques, était plus que sujet à caution, surtout dans l'interprétation qu'en donnait Vigor. Bouju admet évidemment que les décisions d'une assemblée conciliaire, quand elle bénéficie du privilège d'œcuménicité, sont irréformables et s'appliquent à l'ensemble des catholiques, mais en période d'intersession du concile, la papauté assume son rôle oraculaire avec assurance d'inerrance :

«Vigor ne remesle-t-il pas encore derechef le ciel et la terre? Luther, Calvin et les autres hérétiques ennemis de l'Église et de la religion de Dieu ne disent-ils pas de l'Église la mesme chose qu'il dict de l'Église romaine? Elle n'errera point pourveu qu'elle suive la parolle de Dieu. Et qui jugera si en faisant les canons elle aura suivy la parolle de Dieu. Quoy? Luther et Calvin l'examineront, comme Vigor fera de l'Église romaine pour sçavoir si elle a observé les conditions requises à son infaillibilité30. .»

28. Ibid., p. 26.

29. Ibid., p. 81.

30. Ibid., p. 114-115. 
L'interrogation, strictement rhétorique, réitérait habilement les imputations de calvinisme et de luthéranisme adressées à Vigor. En sa doctrine se faisait le procès du richérisme, dénoncé pour hérésie larvée. Bouju rappelle hautement que les attentats contre l'Église catholique commencent toujours par la mise en cause de l'autorité de son chef: "Par quel moyen a-t-on ruiné la religion ès païs septentrionnaux qu'en assaillant le Sainct Siège et tirant droict à la puissance spirituelle du Pape ? Les opinions de Luther, de Calvin et de leurs sectaires ne s'accordent-elles pas pour ce regard en la pluspart des points que Vigor soustient ${ }^{31}$ ? » Bouju se remémorait opportunément l'exemple de l'Angleterre : le schisme avait été causé par la volonté de se soustraire à l'obédience du pontife romain, et l'unité de l'Église avait été mise à mal par la création d'une royauté temporelle qui se prétendait aussi chef ecclésial. La conclusion s'imposait d'elle-même :

« Il paroist clairement par ce que nous avons touché jusques en ce lieu des escrits de Vigor qu'il n'y a autre différence entre luy et les hérétiques en ce qui concerne ceste matière de la puissance spirituelle du pape et hiérarchie, sinon que ce qu'ils nient ouvertement, il le déguise avec quelques parolles affirmatives, et le nie comme eux en effect ${ }^{32}$. »

À l'évidence, le gallicanisme s'était transformé : d'ecclésiologie antiromaine mais catholique, il était devenu hérésie; en poursuivant les abus, il s'était fait dérèglement abusif, franchissant une impalpable limite aux confins de l'hétérodoxie.

La faute en incombe incontestablement au développement outrancier de la polémique autour du pouvoir pontifical au temporel. Bouju en est d'ailleurs parfaitement conscient, puisqu'il éprouve la nécessité de revenir sur les principales étapes de la controverse -ignorant quand même superbement l'Interdit vénitien et l'affaire du serment d'allégeance anglais. À l'en croire, la publication du De potestate papce (1609) du juriste Guillaume Barclay, auquel Bellarmin avait répondu par son De potestate Summi Pontificis, n'avait pas eu lieu d'être,

« attendu qu'il n'y avoit alors aucune cause légitime de resveiller cette question, qui estoit tellement assoupie entre nous depuis que le deffunct Roy Henry le Grand avoit embrassé la religion catholique

31. Ibid., p. 209-210.

32. Ibid., p. 215-216. 
et receu l'absolution de Sa Saincteté qu'il ne restoit point de raison aux François d'estimer qu'aucun de leurs princes donnast subject de l'émouvoir à l'advenir, estans tous catholiques par la grâce de Dieu $»^{33}$.

Richer n'avait pas mieux fait en publiant son De ecclesiastica et politica potestate, et Simon Vigor, d'après Bouju, a fait pis que les deux autres dans son Apologia contre André Duval. Puis est venu De Dominis, qui « a pris la hardiesse de publier le ramas d'erreurs et hérésies contenuës en son livre contre Sa Saincteté et la hiérarchie de l'Église pour leur ayder à l'affliger $»^{34}$. Le De Republica ecclesiastica de l'ancien archevêque de Spalato était l'ultime témoignage d'une communauté de sensibilité qui regroupait des antiromains prétendument catholiques, mais réellement hérétiques.

\section{Les thèses de Vigor et L'eCCLÉSiologie de De Dominis}

Le rapprochement était perfide, il compromettait la doctrine vigorienne avec l'enseignement d'un apostat, mais il n'était pas sans fondement. À lire les Quatre livres de l'Estat et gouvernement de l'Église publiés en 1621 par Vigor contre la Défense de Bouju, la comparaison semble spontanément nécessaire avec les principales thèses du De Républica ecclesiastica, et notamment nombre de ses propositions que la Faculté de théologie de Paris avait condamnées le 15 décembre 1617. Dans son livre de 1621, Vigor accuse le Saint-Siège d'être la cause première de la controverse écrite qui dure depuis plus d'une décennie. Il souligne le fait que les princes qui souhaitent développer indûment leur puissance ne manquent jamais de recourir à des écrivains pour conférer légitimité à leurs scandaleuses prétentions :

« C'est l'artifice dont souvent s'est servi la cour de Rome en introduisant tant de nouvelles religions pour avoir des escrivains et des trompettes ès prédications, pour publier et auctoriser l'érection de ce nouvel autel de puissance absoluë du Pape, à l'encontre de l'auteur sacré et inviolable de l'auctorité souveraine de l'Église, lesquelles Religions, outre leur intérest général avec les autres ecclésiastiques, touchant l'exemption prétenduë de la justice séculière, pour avoir plus de liberté

33. Ibid., p. 228.

34. Ibid., p. 229. 
de parler au mespris de l'Estat séculier, sont d'ailleurs intéresséez et engagéez à ceste nouvelle doctrine de la puissance absoluë pour maintenir leurs illégitimes establissemens ${ }^{35}$. »"

Vigor admet la primauté romaine dans l'Église, mais il nie que du primat découle puissance absolue sur la communauté ecclésiale. Une fois encore, Vigor récuse le fait que le pape soit chef essentiel, il en reste au statut ministériel, selon une perspective rigoureusement gallicane.

Le gallicanisme richériste et vigorien n'a pas manqué d'adversaires qui, à l'exemple de Bouju, mettaient en cause, et vigoureusement, l'orthodoxie catholique de ses tenants. Outre Bouju, Vigor a distingué le jésuite Louis Richeome (1544-1625), qui avait publié à Caen en 1615 ses Advis et notes sur quelques plaidoyers de M. Louis Servin, advocat du Roy, ci-devant publiés en France au préjudice de la religion catholique, de l'honneur du Roy Très-Chrestien et de la paix de son royaume - le titre indiquait sans ambiguïté que même Servin était désormais tenu pour ennemi du catholicisme. D'où la nécessité, pour Vigor, de commencer par une infrangible profession d'appartenance confessionnelle :

«La grâce que je reçoy de Dieu d'estre catholique et l'honneur que j'ay d'estre François et officier du Roy m'ont obligé par cy-devant de descouvrir l'abus de plusieurs escrivains de ce siècle, qui soubs un faux prétexte de religion, veulent changer le gouvernement aristocratique de l'Église en une souveraineté absoluë et par ce moyen abolir la liberté chrestienne pour nous réduire en servitude et soubs ombre de défendre l'authorité légitime de nostre sainct Père le Pape, renverser de fond en comble les puissances royales et séculières ${ }^{36}$. »

Du livre de Richeome, Vigor ne retient qu'un ramassis d'erreurs et d'opinions fallacieuses. Il accuse le jésuite de vouloir rétablir une théocratie qui n'est que le symétrique, non moins fautif, du régime césaropapiste défendu par le marsilisme :

« Il bande tout son esprit à monstrer que le Pape est monarque très absolu de toute la Chrestienté, tant au spirituel que temporel, et résoult totalement l'authorité politique en la puissance ecclésiastique, tout ainsi que jadis Marsilius de Padoue confondoit l'ecclésiastique en la politique, qui sont deux erreurs contraires, lesquelles on doit également

35. S. Vigor, Quatre livres de l'Estat et gouvernement de l'Église, op. cit., p. 15.

36. Ibid., p. 41-42. 
éviter et se tenir à la parole de Dieu, qui a estably l'ecclésiastique sur le spirituel et la séculière sur le temporel ${ }^{37}$. »

Vigor entreprend de véhémentes vociférations contre l'introduction de l'idée d'une monarchie pontificale absolue dans l'Église. À l'en croire, le mal a commencé lorsque le patrimoine de saint Pierre s'est accru sous les règnes de Pépin le Bref et de Charlemagne,

« tellement que depuis ce temps-là, pour mieux fonder la puissance temporelle du Pape, les flatteurs ont employé la spirituelle, jusques à luy arroger une authorité sur les Roys et autres princes de la Chrestienté, doctrine totalement contraire à la loy de Grâce en laquelle nostre Seigneur enseigne que les Roys seigneurient et commandent par un pouvoir absolu $»^{38}$.

Au contraire, les prélats de l'Église n'ont de pouvoir que ministériel - rien, donc, de coactif. Anti-absolutistes, les catholiques devaient pieusement l'être, et Vigor retrouvait là une idée essentielle aux ecclésiologies de Richer, de Sarpi et de De Dominis : «Ce seroit chose de trop grande conséquence que nostre salut fust en la disposition absoluë d'un homme, subject aux passions lesquelles offusquent ordinairement le jugement et empeschent de pouvoir exactement discerner ce qui est de la justice ${ }^{39}$. » L'Écriture n'enseigne pas qu'il y ait puissance sur terre, ecclésiastique ou non, à qui les princes temporels doivent rendre compte, argument supplémentaire à l'encontre de la moderne théocratie que désirent réaliser les thuriféraires aveuglés de la papauté absolutiste.

L'idée aristocratique demeurait le fondement d'une ecclésiologie catholique antiromaine qui voulût exorciser par avance le reproche d'hétérodoxie. Le précédent de Pierre, aigrement repris par saint Paul, était opportunément rappelé. En l'Épître aux Galates $(2,11)$, il est raconté que saint Pierre s'étant laissé aller à une dissimulation contre la vérité de l'Évangile, Paul l'avait vertement tancé : «Or Céphas étant à Antioche, je lui résistai en face parce qu'il était répréhensible. » Pour Vigor, la conclusion était évidente : résistance était permise à l'égard du souverain pontife si on ne voulait pas le faire plus grand que saint Pierre; de surcroît, la figure de saint Paul

37. Ibid., p. 42-43.

38. Ibid., p. 47-48.

39. Ibid., p. 55-56. 
égalait le prince des Apôtres. De souveraineté de Pierre, il n'avait jamais été question :

«Aussi nostre Seigneur qui a estably les Roys pour juger son peuple est luy mesme qui a commandé de rapporter les plainctes des chrestiens, non à un monarque absolu, comme il faict aux princes séculiers, mais au conseil aristocratique de l'Église, lequel à ceste fin il establit ${ }^{40}$.»

Saint Pierre n'a jamais rien entrepris d'autorité privée; il semble au contraire qu'au temps de l'Église primitive, chaque décision ait été rapportée en commun pour être prise par la communauté des Apôtres. Le pouvoir des clefs a été remis au groupe apostolique en tant qu'il représentait l'Église. Comme lui, le pape n'a qu'une puissance ministérielle; il ne peut certes pas revendiquer de pouvoir in rebus temporalibus, même indirect. Vigor le rappelle avec force :

"Soubs prétexte d'hérésie, apostasie, excommunication, ou aucune autre sentence que le Pape pourroit donner contre les Roys, les subjects ne sont jamais absous du serment de fidélité, ains leur doivent toute sorte de submission et obéïssance, sinon aux choses contraires aux commendements de Dieu, et sont tenus de les recognoistre comme leurs Roys légitimes, tels qu'il plaist à Dieu nous les donner bons selon sa clémence et miséricorde, mauvais selon nos péchéz et démérites ${ }^{41}$. »

De rien ne servait au pontife romain d'invoquer les religieuses franchises de son vicariat christique. Le Livre du prophète Jérémie $(27,5)$ attribue franchement à Dieu l'institution des princes temporels parce qu'il est le créateur de la terre : «C'est moi qui ait créé la terre par ma grande puissance et par mon bras fort, et j'ai donné la terre à qui il m'a plu. » Pour Vigor, l'argument est sans appel contre un recours aux privilèges du vicariat en faveur du pouvoir indirect, car ce qui découle de la qualité de créateur est incommunicable aux vicaires de Dieu : « Donc ce qui vient en conséquence de la création est incommunicable au Pape. C'est donc à Dieu seul, comme Dieu, roy des roys et comme créateur, d'instituer et déposer les roys ${ }^{42}$. » $\mathrm{Se}$ dénonçait continûment la lourde responsabilité du Saint-Siège dans un dévoiement doctrinal qui défigurait l'Église. Rome, unique objet du ressentiment de Vigor, Rome, enfin, qui avait fait taire la voix

40. Ibid., p. 64.

41. Ibid., p. 168.

42. Ibid., p. 170. 
du concile pour mieux imposer l'assourdissante déclamation de son oracle mensonger. Pour empêcher les assemblées conciliaires, on a représenté, explique Vigor, les difficultés de les réunir,

«mais en un mot, la seule cour de Rome est cause de tous ces désordres, car prévoyant que si les évesques s'assembloient souvent en concile, le gouvernement aristocratique petit à petit se restabliroit et que l'Église viendroit finalement à recognoistre le juste pouvoir qu'elle tient immédiatement de Dieu et que la monarchie absoluë infaillible s'esvanoüyroit, elle fomente et entretient fort soigneusement les divisions et partialitéz entre les Princes Chrestiens $»^{43}$.

En une ultime bravade, Vigor rappelle qu'aux termes des canons de Constance et de Bâle, les conciles légitimement assemblés bénéficient de l'assistance du Saint-Esprit; l'infaillibilité de leurs décisions leur est garantie, et ils n'ont que faire de la confirmation du souverain pontife.

Avec la naissance de la papauté moderne, l'âge posttridentin a connu une mutation majeure dont la capacité de bouleversement est encore imparfaitement mesurée par l'historiographie. En 1960, Alphonse Dupront ne craignait pas de l'affirmer : " Trente fonde l'absolutisme romain par des apports qui lui appartiennent en propre $^{44}$. » L'historien du Mythe de croisade précisait :

«La catholicité romaine commence à Trente, et cette monarchie spirituelle où Rome va définir au long des siècles modernes, avec les principes et les formes d'un absolutisme temporel, l'extraordinaire présence d'une puissance quasi uniquement spirituelle, et une unité de religion. De cette unité, comme de la puissance d'universel, la caution et le signe demeurent le pontife romain et Rome capitale. Ils sont les serviteurs du règne, au centre d'une monarchie unitaire qui ramène à soi toute l'Église ${ }^{45}$.»

Là était bien le problème à quoi se sont tenacement affrontés les gallicans richéristes : l'impérieuse volonté unifiante d'une papauté dominatrice et corroborée par les récents acquis conciliaires. Curieusement, le centre de gravité de la dispute n'est plus tant la question de la potestas indirecta que celle de la légitimité d'une monarchie absolue dans l'Église, comme si la première dépendait étroitement

43. Ibid., p. 453.

44. Alphonse Dupront, «Le concile de Trente», Le concile et les conciles. Contribution à l'histoire de la vie conciliaire de l'Église, Paris-Chevetogne, 1960, p. 195-243, repris dans id., Genèses des Temps modernes. Rome, les Réformes et le Nouveau Monde, éd. D. Julia et Ph. Boutry, Paris, 2001, p. 173-206 [p. 202].

45. Ibid., p. 201. 
de la seconde. Par un singulier déplacement du débat, le richérisme en est venu à développer un enseignement riche de virtualités politiques qui ont probablement soutenu la royauté dans son projet de se désolidariser d'une mouvance qui se faisait par ailleurs de plus en plus compromettante sur un plan strictement confessionnel. L'idée aristocratique était le fondement cardinal à partir de quoi s'ordonnait l'ensemble du discours tenu par le De Republica ecclesiastica de De Dominis. L'ancien archevêque de Spalato y définissait un épiscopalisme fondé sur une collégialité absolue : «De même que les Apôtres ont dirigé ensemble et aristocratiquement l'Église avec puissance égale et universelle, de même les évêques sont-ils chacun à la tête de l'Église avec pleine puissance ${ }^{46}$. » Le 15 décembre 1617, la Faculté de théologie de Paris avait condamné la proposition comme hérétique, schismatique et unitatis Ecclesice subuersiua; pleinement hérétique également, la thèse qui déclarait que la doctrine de Jean Hus, selon quoi l'Église ne connaît pas d'autre chef suprême et monarque que le Christ, est chrétienne et confirmée par les écrits des Pères. Enseignement qui n'était vraiment pas éloigné de celui de Simon Vigor. Le richérisme vigorien s'est certes tenu à l'écart des outrances théologiques de De Dominis, il n'en a pas moins proclamé des thèses ecclésiologiques comparables, ignorant sans vergogne les anathèmes lancés par la Sorbonne. Alors que nombre de gallicans, après la semonce de novembre 1617, ont préféré rentrer dans le rang, Simon Vigor ranime en 1621 la flamme d'un débat lancé en 1611 par la publication du De ecclesiastica et politica potestate de Richer; il représente désormais les intérêts d'un gallicanisme radical et obstiné qui refuse de faire les frais du discrédit jeté sur l'antiromanisme catholique par le revirement récent des docteurs parisiens.

sylvio.de-franceschi@laposte.net

46. Cité dans Censura Sacrce Facultatis Theologice Parisiensis in quatuor libros De Republica ecclesiastica, auctore Marco Antonio De Dominis, Archiepiscopo Spalatensi, Paris, 1618, repris dans C. Duplessis d'Argentré, Collectio iudiciorum de nouis erroribus, qui ab initio duodecimi seculi post incarnationem Verbi usque ad annum 1632, in Ecclesia proscripti sunt et notati, 3 t. en 3 vol., Paris, 17281736, t. II, $2^{\mathrm{e}}$ partie, p. 103-109, censure 14, lib. II, cap. I, num. 9 : « Sicut Apostoli simul et insolidum aristocratice curam gerebant Ecclesice cum potestate cequali et uniuersali, ita episcopi omnes simul et insolidum eandem regunt Ecclesiam, singuli cum plena potestate. » 